\section{Le code de localisation des ARNm se révèle}

Eric Lécuyer

University of Toronto,

Terrence Donnelly Centre for Cellular

and Biomolecular Research,

University of Toronto, 160 College Street,

Room 504, Toronto, Ontario, M5S $3 \varepsilon 1$ Canada.

eric.lecuyer@utoronto.ca

\section{Localisation des protéines \\ dans la cellule}

La cellule répartit ses composants au sein d'un vaste réseau de compartiments intracellulaires, dont chacun est équipé d'un assemblage spécifique de protéines remplissant des fonctions structurales et catalytiques précises. La vision traditionnelle suggère que la localisation des protéines est principalement déterminée après leur synthèse, grâce à des séquences peptidiques intrinsèques reconnues par des machineries de localisation spécialisées. Une alternative existe, qui implique la localisation des transcrits d'ARN messagers (ARNm), avant que ceux-ci ne soient traduits par les ribosomes [1-3]. Ce processus offre plusieurs avantages pour la cellule. D'abord, puisqu'une molécule d'ARNm peut être traduite à plusieurs reprises, il s'agit d'un moyen efficace pour économiser l'énergie requise pour acheminer individuellement chaque protéine vers sa destination finale. De plus, cela permet de prévenir la localisation aberrante de protéines à des endroits où elles pourraient avoir des effets néfastes, voire mêmes toxiques, pour la cellule et l'organisme.

\section{Localisation subcellulaire des ARNm}

Au cours des deux dernières décennies, des ARNm localisés ont été impliqués dans une variété de processus biologiques associés à la polarisation cellulaire, incluant la formation de gradients morphogénétiques, la spécification des axes embryonnaires, la division cellulaire asymétrique, et la migration cellulaire [1-4]. Malgré ces nombreux exemples, il est communément admis que la localisation des ARNm à un endroit restreint de la cellule représente un phénomène rare et limité à des transcrits spécialisés. En règle générale, la localisation d'un transcrit est contrôlée via des séquences régulatrices en cis présentes dans les ARNm, situées souvent dans la région 3' non traduite, et qui sont spécifiquement reconnues par des complexes ribonucléoprotéiques (RNP) chargés de transporter les ARNm correspondants [5]. Trois mécanismes principaux de localisation des ARNm ont été caractérisés: le transport dirigé sur les composants du cytosquelette (microtubules ou filaments d'actine), la diffusion passive suivie d'un ancrage dans une région précise de la cellule, et l'existence de mécanismes protégeant localement des transcrits qui sont dégradés dans le reste de la cellule $[6,7]$. Malgré ces connaissances, dans la plupart des cas les éléments en cis et la machinerie moléculaire régulant le ciblage de transcrits spécifiques restent à identifier.

\section{Analyse systématique} de la distribution des transcrits cellulaires: révélations inattendues Jusqu'à récemment, aucune étude n'avait permis une analyse systématique des caractéristiques de localisation subcellulaire des ARNm, et ce en raison essentiellement de limitations dans la résolution des techniques de marquage disponibles. Cela expliquait pourquoi la diversité globale des patrons de localisation des ARNm et leur prévalence à l'échelle génomique demeuraient inconnues. Afin d'explorer ces questions, nous avons optimisé une méthode d'hybridation in situ utilisant la fluorescence et permettant une visualisation à haute résolution de la distribution subcellulaire des transcrits géniques [8]. Cette approche fut ensuite appliquée d'une manière systématique, afin de caractériser la dynamique de localisation des ARNm codés par 3370 gènes distincts ( $25 \%$ du génome) durant l'embryogenèse chez la mouche à fruit, Drosophila melanogaster [8]. Les résultats de cette étude, comprenant des images d'embryons à différents stades du développement et une description des caractéristiques de localisation pour chaque ARNm analysé, ont ensuite été organisés dans une base de données accessible au public (http://fly-fish. ccbr.utoronto.ca/), afin de faciliter l'analyse des données et d'offrir une ressource utile à la communauté scientifique.

Plusieurs conclusions surprenantes émergent de cette étude. Tout d'abord, la grande sensibilité de la technique utilisée a permis de révéler que les ARNm dans leur majorité $(71 \%)$ se distribuent de façon localisée au cours du développement embryonnaire [8], remettant complètement en cause les prédictions précédentes ( $1 \%$ d'ARNm répartis de façon localisée) établies chez cet organisme [9]. Nous avons identifié une quarantaine de patrons de localisation majeurs, incluant des phénotypes d'exclusion où les transcrits sont spécifiquement exclus d'une région 
particulière de la cellule, ainsi qu'une variété de distributions plus fines en association à différentes structures cellulaires/organites (Figure 1) [8]. L'analyse bioinformatique des résultats a ensuite démontré que la majorité des catégories de localisation qui ont été définies sont spécifiquement enrichies pour des transcrits ayant des fonctions biologiques apparentées, suggérant que ce processus de répartition des transcrits joue un rôle central dans l'organisation fonctionnelle des réseaux subcellulaires, et que les phénotypes de localisation ont une utilité importante dans la prédiction des fonctions géniques.

\section{Le code de localisation des ARNm :} ordonnateur des fonctions cellulaires? Compte tenu de la fréquence et de la variété des événements observés, l'implication majeure de cette étude est qu'il existe un vaste réseau de régulation post-transcriptionnelle que l'on

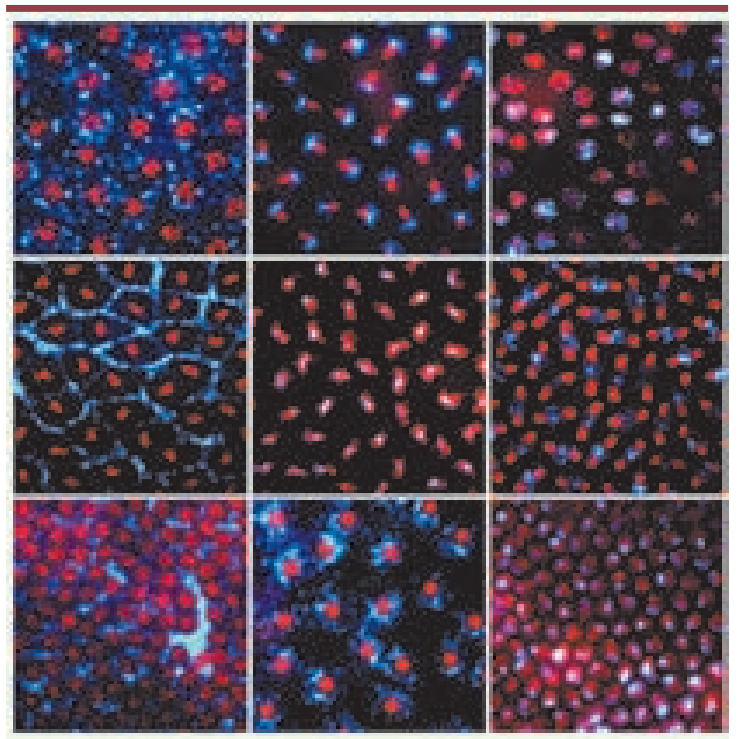

Figure 1. Mosaïque de patrons de localisation de transcrits d'ARNm chez les embryons de D. melanogaster. Ces images de la surface embryonnaire illustrent la diversité des patrons de distribution caractérisés, incluant des transcrits localisés dans des domaines cytoplasmiques, sur l'appareillage mitotique, dans des domaines nucléaires/chromatiniens et au niveau des jonctions cellulaires. Les ARNm sont illustrés en bleu et l'ADN des noyaux en rouge. pourrait désigner comme le «code de localisation des ARNm». Puisque la localisation d'un transcrit précède généralement sa traduction en protéine, ce code régulateur jouerait un rôle central dans l'assemblage localisé d'une variété de complexes macromoléculaires. Les exemples de processus vraisemblablement régulés par des ARNm localisés incluent la régulation de voies de signalisation, de l'adhésion cellulaire, de la polarité cellulaire, du cytosquelette, de la structure nucléaire/ chromatinienne, et de la machinerie mitotique. Une autre possibilité séduisante serait que certains ARNm localisés exerceraient des fonctions structurales ou catalytiques indépendantes de leur fonction codante. Par exemple, ces molécules pourraient représenter des sous unités fonctionnelles de complexes RNP, comme on en observe avec les composantes ARN des ribosomes ou d'autres assemblages macromoléculaires [10]. L'ensemble de ces possibilités ne devrait peut-être pas nous surprendre si l'on en croit l'hypothèse selon laquelle les ARN auraient précédé les protéines dans l'évolution des fonctions cellulaires [11].

\section{En conclusion}

Cet article et la ressource web qui l'accompagne serviront de point de départ pour de nombreuses études à venir visant à déchiffrer le code de localisation des ARNm et l'étendue de son impact pour la régulation de l'expression génique et de la physiologie cellulaire. La conservation des réseaux géniques et des processus cellulaires entre la drosophile et les vertébrés suggère que ce code de localisation des
ARNm sera aussi hautement conservé chez les eucaryotes supérieurs. On peut d'ailleurs suspecter que l'évolution des voies de localisation des ARNm ait joué un rôle important dans l'évolution de la complexité phénotypique des organismes. Élucider les mécanismes de localisation des $A R N m$, et potentiellement d'autres familles d'ARN non codants, s'avère une voie d'investigation importante afin de comprendre les principes régissant l'organisation cellulaire normale et leur défaillance dans différents contextes pathologiques. $\diamond$

Unraveling the mRNA localization code

\section{REMERCIEMENTS}

Ce travail fut accompli avec l'appui des Instituts de Recherche en Santé du Canada. J'aimerais remercier le Dr Henry Krause pour son soutien, ainsi que les Drs Gwenael BadisBréard et Mathieu Gabut pour leurs commentaires sur ce manuscrit.

\section{RÉFÉRENCES}

1. St Johnston D. Moving messages : the intracellular localization of mRNAs. Nat Rev Mol Cell Biol 2005; $6: 363-75$.

2. Jansen RP. mRNA localization: message on the move. Nat Rev Mol Cell Biol 2001 ; 2 : 247-56.

3. Kloc M, Zearfoss NR, Etkin L. Mechanisms of subcellular mRNA localization. Cell 2002; 108 : 533-44.

4. Giet $R$, Prigent $C$. Contrôle de la détermination cellulaire par les centrosomes. Med Sci (Paris) 2003; $19: 656-8$.

5. Tekotte H, Davis I. Intracellular mRNA localization: motors move messages. Trends Genet 2002 ; $18: 636-42$.

6. Lipshitz HD, Smibert CA. Mechanisms of RNA localization and translational regulation. Curr Opin Genet Dev 2000; $10: 476-88$.

7. Czaplinski K, Singer RH. Pathways of mRNA localization in the cytoplasm. Trends Biochem Sci 2006 ; $31:$ 687-93.

8. Lécuyer $\varepsilon$, Yoshida $H$, Parthasarathy $N$, et al. Global analysis of mRNA localization reveals a prominent role in organizing cellular architecture and function. Cell $2007 ; 131: 174-87$.

9. Tomancak P, Beaton A, Weiszmann R, et al. Systematic determination of patterns of gene expression during Drosophila embryogenesis. Genome Biol 2002 ; 3 (online).

10. Prasanth KV, Spector DL. Eukaryotic regulatory RNAs : an answer to the 'genome complexity' conundrum. Genes Dev 2007 ; 21 : 11-42.

11. Gilbert W. The RNA world. Nature 1986 ; 319 : 618. 